providers in the identification and management of patients found to be at risk.

Disclosure: No significant relationships.

Keywords: medical settings; Suicide; Screening; suicide risk

\section{Training in psychiatry}

\section{O308}

\section{5 years follow up study on changes of romanian} psychiatric residents' opinion on factors which influence their decision to emigrate

\author{
A. Mihai ${ }^{1 \star}$, S. Trandafir ${ }^{2}$, L. Duica ${ }^{3}$, A. Mihai ${ }^{4}$, C. Lungu $^{5}$ and \\ C. Pirlog ${ }^{6}$ \\ ${ }^{1}$ Me2, GE PALADE University of Medicine, Pharmacy, Science and \\ Technology of Târgu Mure, Târgu Mureș, Romania; ${ }^{2}$ Psychiatry, Carol \\ Davila University of Medicine and Pharmacy, Bucharest, Romania; \\ ${ }^{3}$ Psychiatry, Lucian Blaga University, Sibiu, Sibiu, Romania; \\ ${ }^{4}$ Psychiatry, Iuliu Hatieganu University of Medicine and Pharmacy of \\ Cluj Napoca, Cluj Napoca, Romania; ${ }^{5}$ Statistics, Spiru Haret University \\ of Bucharest, Bucharest, Romania and ${ }^{6}$ Sociology, University of \\ Medicine and Pharmacy of Craiova, Craiova, Romania \\ ${ }^{*}$ Corresponding author. \\ doi: 10.1192/j.eurpsy.2021.474
}

Introduction: Important changes have been done in economic status of residents in 2018. The impact of these measures in changing opinion was checked.

Objectives: The prioritization exercises of main factors related with psychiatric residents' decision to emigrate could be a starting point of elaboration of a strategy of reforms.

Methods: The study was cross sectional evaluation at national level on a randomized selected sample of Romanian psychiatric residents' opinion on factors which influence decision of migration in EU countries in two time points: 2015 and 2020.

Results: $38 \%$ of residents intend to work abroad comparing with $78 \%$ before the economic changes $(25.84 \%$ versus $71.66 \%$ for a limited period of time and $15.73 \%$ versus $28.33 \%$ intend to emigrate) and $2 \%$ versus $5 \%$ intend to leave the speciality. The most important factors for decision to emigrate changed from "Better working conditions" (15.73 versus $37.31 \%$ residents) to "Better training" $20.25 \%$ residents and the factor "respect and appreciation by colleagues" remained important for $19.10 \%$ versus $17.91 \%$. "Lack of working place for partner" was considered by $26.96 \%$ of responders as an important disadvantage of working abroad. "Being far from family members" which was considered 5 years ago by $64.18 \%$ of responders as an important disadvantage of working abroad, nowadays concerns only $6.74 \%$, probably because it seems easier to go abroad together with the family members.

Conclusions: The factors (better training in psychiatry and psychotherapy, better supervision, more involvement in research) which influence the residents' decision to emigrate represent the starting points on futures reforms in educational and medical system in psychiatry.

Disclosure: No significant relationships.

Keywords: emigration; mental health; residents; training
Women, gender and mental health

\section{9}

Association between hair loss severity and risk for later mental health problems in women irradiated for tinea capitis in childhood

D. Segal-Engelchin ${ }^{1 \star}$ and S. Shvarts ${ }^{2}$

${ }^{1}$ Social Work, Ben-Gurion University of the Negev, Beer-Sheva, Israel and ${ }^{2}$ Faculty Of Health Sciences, Ben-Gurion University of the Negev,

Beer Sheva, Israel

${ }^{\star}$ Corresponding author.

doi: 10.1192/j.eurpsy.2021.475

Introduction: Hair loss resulting from childhood irradiation for tinea capitis has been linked to mental health effects in women. However, the association of hair loss severity with mental health in this population is unknown.

Objectives: The aim of this study is to examine the association between hair loss severity and mental health outcomes in women irradiated for tinea capitis in childhood and to identify contributing factors to these outcomes.

Methods: Medical records, held at the archives of Israel National Center for Compensation of Scalp Ringworm Victims, were retrospectively reviewed for 2509 women who received compensation for full or partial alopecia resulting from irradiation in childhood for tinea capitis. Mental health outcomes were determined by the number of mental health conditions reported.

Results: Among women with high hair loss levels, risk was increased for a range of mental health problems, including depression symptoms, emotional distress, social anxiety, low self-esteem, and suicidal ideation. Hair loss severity emerged as a significant predictor of mental health, adding to the effects of other predictors such as family, and social and physical health problems. Effects of hair loss severity on mental health outcomes were mediated by women's negative social experiences.

Conclusions: Hair loss severity is a significant risk factor for mental health problems in women irradiated for tinea capitis in childhood. Further research is needed to assess mental health risks among women with severe hair loss associated with additional diseases.

Disclosure: No significant relationships.

Keywords: hair loss severity; mental health problems; irradiation treatment; women

\section{$\mathrm{O} 310$}

\section{Restraint theory: Significance of rumination}

A. Brytek-Matera ${ }^{1 \star}$, P. Bronowicka ${ }^{2}$ and J. Walilko ${ }^{2}$

${ }^{1}$ Katowice Faculty Of Psychology, SWPS University of Social Sciences and Humanities, Katowice, Poland and ${ }^{2}$ Institute Of Psychology,

University of Wroclaw, Wroclaw, Poland

${ }^{\star}$ Corresponding author.

doi: 10.1192/j.eurpsy.2021.476

Introduction: Restraint theory (Herman and Polivy, 1975) suggests that human eating behaviour is under cognitive control and this leads to reduced sensitivity to internal cues for satiety, resulting in overeating in situations where cognitive control is under-mined (Johnson et al., 2012). In other words, restraint theory suggests that 